
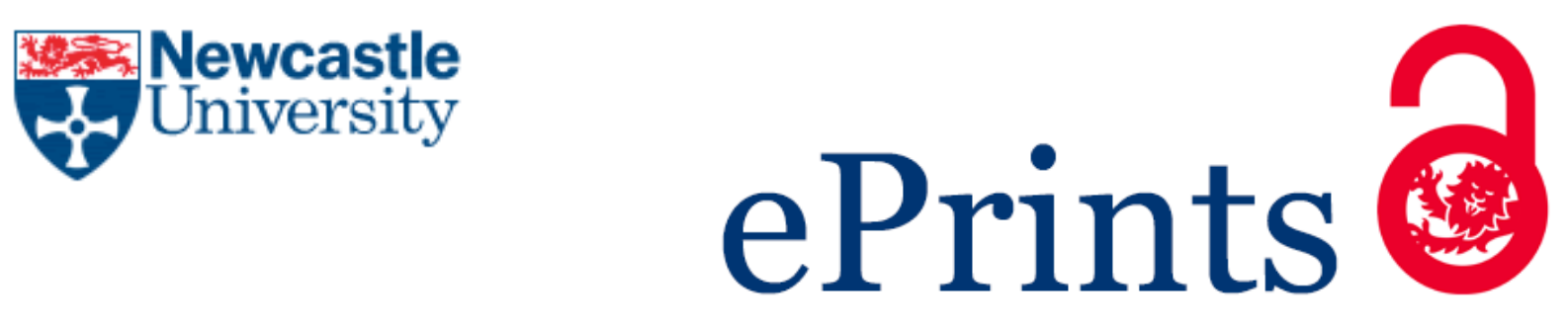

Crossland KL, Abinun M, Arkwright PD, Cheetham TD, Pearce SH, Hilkens $\mathrm{CMU}$, Lilic D. AIRE is not essential for the induction of human tolerogenic dendritic cells. Autoimmunity 2016, 25, 1-8.

\title{
Copyright:
}

This is an Accepted Manuscript of an article published by Taylor \& Francis in Autoimmunity on 25/02/2016, available online: http://www.tandfonline.com/10.3109/08916934.2016.1148692.

DOI link to article:

http://dx.doi.org/10.3109/08916934.2016.1148692

Date deposited:

$15 / 03 / 2016$

Embargo release date:

25 February 2017

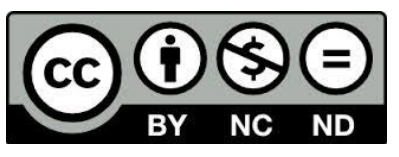

This work is licensed under a

Creative Commons Attribution-NonCommercial-NoDerivatives 4.0 International licence 


\section{AIRE is not essential for the induction of human tolerogenic} dendritic cells GAUT-2015-0082 (R2) Katherine L Crossland ${ }^{1}$, Mario Abinun ${ }^{1,2}$, Peter D Arkwright ${ }^{3}$, Timothy D Cheetham ${ }^{4}$, Simon
H Pearce ${ }^{5}$, Catharien M U Hilkens ${ }^{6}$, Desa Lilic

${ }^{1}$ Primary Immune Deficiency Group, Institute of Cellular Medicine, Newcastle University, UK

${ }^{2}$ Department of Paediatric Immunology, Great North Children's Hospital, Newcastle upon Tyne, UK

${ }^{3}$ Institute of Inflammation and Repair, University of Manchester

${ }^{4}$ Department of Paediatric Endocrinology, Great North Children's Hospital, Newcastle upon Tyne, UK

${ }^{5}$ Institute of Human Genetics, Newcastle University, UK

${ }^{6}$ Musculoskeletal Research Group, Institute of Cellular Medicine, Newcastle University, UK

${ }^{7}$ Regional Immunology and Allergy Department, Newcastle upon Tyne Hospitals NHS Trust, UK

Key Words

AIRE; APS1; APECED; tolerogenic; dendritic cells, monocyte-derived dendritic cells

Correspondence to:

Desa Lilic MD MSc DSc/PhD FRCPath

Institute of Cellular Medicine

The Faculty of Medical Sciences

Newcastle University

NE2 4HH 9

United Kingdom

Tel ++ 001912087244

Fax ++ 001012085455

Email: desa.lilic@ncl.ac.uk 
1 Abbreviations

2 APS - Autoimmune Polyendocrinopathy Syndrome type I

3 APECED - Autoimmune Polyendocrinopathy Candidiasis Ectodermal Dystrophy

4 AIRE - Autoimmune Regulator

5 DC - dendritic cell

6 moDC - monocyte-derived DC

7 tolDC - tolerogenic moDC

8 inflDC - inflammatory moDC

9 mTECs - medullary thymic epithelial cells

10 eTACs - extrathymic AIRE expressing cells

11 CMC - chronic mucocutaneous candidiasis

12 PBMC - peripheral blood mononuclear cells

13 Dex - dexamethasone

14 VitD3 - calcitrol

15 APC - antigen presenting cell

16 MHC - major histocompatibility complex

17 PDL-1 - programmed death receptor ligand 1

18 TLR - toll like receptor

19 
1

2

3

4

5

6

7

8

\section{Summary}

Loss-of-function mutations of the Autoimmune Regulator (AIRE) gene result in organspecific autoimmunity and disease Autoimmune Polyendocrinopathy type 1 (APS1) / Autoimmune Polyendocrinopathy Candidiasis Ectodermal Dystrophy (APECED). The AIRE protein is crucial in the induction of central tolerance, promoting ectopic expression of tissuespecific antigens in medullary thymic epithelial cells, enabling removal of self-reactive Tcells. AIRE expression has recently been detected in myeloid dendritic cells (DC), suggesting AIRE may have a role in peripheral tolerance. DC stimulation of T-cells is critical in determining the initiation or lack of an immune response, depending on the pattern of costimulation and cytokine production by DCs, defining immunogenic/inflammatory (inflDC) and tolerogenic (tolDC) DC. In AIRE-deficient patients and healthy controls we validated the role of AIRE in the generation and function of monocyte-derived inflDC and tolDCs by determining mRNA and protein expression of AIRE and comparing activation markers (HLA-DR/DP/DQ,CD83,CD86,CD274(PDL-1),TLR-2), cytokine production (IL-12p70,IL10,IL-6,TNF- $\alpha$,IFN- $\gamma$ ) and T-cell stimulatory capacity (mixed lymphocyte reaction) of AIRE+ and AIRE- DCs. We show for the first time that: 1) tolDCs from healthy individuals express AIRE; 2) AIRE expression is not significantly higher in tolDC compared to inflDC; 3) tolDC can be generated from APECED patient monocytes and that 4) tolDCs lacking AIRE retain the same phenotype and reduced T-cell stimulatory function. Our findings suggest that AIRE does not have a role in the induction and function of monocyte-derived tolerogenic DC in humans, but these findings do not exclude a role for AIRE in in peripheral tolerance mediated by other cell types. 


\section{Introduction:}

The autoimmune regulator (AIRE) is an important regulator of autoimmunity promoting central tolerance in the thymus. AIRE is predominantly expressed in thymic medullary epithelial cells (mTECs), where it promotes the expression of ectopic tissuespecific antigens $(1,2)$, ensuring the deletion of self-reactive T-cells thus ensuring immunological tolerance by preventing autoimmunity.

Over 60 autosomal-recessive, loss-of-function mutations within the AIRE gene in humans described to date underlie the very rare autosomal recessive disease Autoimmune Polyendocrinopathy Syndrome type 1 (APS1), also called Autoimmune Polyendocrinopathy Candidiasis Ectodermal Dystrophy (APECED) (OMIM 240300) (3-5). APS1 is typically characterised by a triad of symptoms including hypoparathyroidism, autoimmune adrenal insufficiency and chronic mucocutaneous candidiasis (CMC) although other autoimmunity, both endocrine (diabetes type 1, ovarian, testicular) and non-endocrine (autoimmune hepatitis) is frequently present. The autoimmunity is consistent with our understanding of the role of AIRE in the removal of self-reactive T-cells in the thymus but the susceptibility of APS1 patients to CMC remained elusive and intriguing until recently, when we (6) and others (7) reported the presence of neutralizing auto-antibodies to interferons type I and Th-17 cytokines (IL-17A, IL-17F and IL-22) in APS1 patient sera. These cytokines are critical for the protection against fungal infections and neutralizing autoantibodies to these cytokines elegantly explain the selective susceptibility to fungal infections in APS1 patients in the context of their autoimmunity. Importantly, the expression of these cytokines, specifically IFN $\alpha$, is not restricted to AIRE-expressing mTECS in the thymus (8) suggesting that AIRE may have additional roles in the periphery in preventing autoimmunity.

The expression of AIRE outside the thymus has been recognised in a number of human tissues including lymph nodes $(9,10)$, spleen (10), PBMC (11) and in monocyte- 
1 dendritic cell lineages (11-14). However, although AIRE gene expression in the periphery is undisputed, detection of AIRE protein has been and still is far more controversial, especially in humans (15) (16).

The role of AIRE in the periphery has been confirmed in a number of Aire deficient ("knock-out") mouse models, where it was shown that Aire is involved in the activation/regulation of T-cells. It was shown that splenic and lymph node Aire ${ }^{-/-}$DC activate naive T-cells more efficiently than do Aire ${ }^{+/+}$DC (17); a splenic population of extrathymic Aire-expressing cells (eTACs), which were shown to be a bone marrow-derived antigenpresenting cell (APC) population, could directly functionally inactivate CD4+ T-cells (18); more recently, AIRE was shown to be directly involved in the perinatal generation of regulatory T-cells crucial for maintain self-tolerance (19). These data indicated that at least in the mouse model, AIRE may have a regulatory/tolerogenic role in the periphery.

The relevance of findings in AIRE deficient mouse models to the human APS1/APECED disease has often been questioned as, although Aire-/- mice develop autoimmunity, they do not develop disease and the repertoire of autoantigens in human disease and mouse models is poorly replicated $(4,20)$. To date, there is very little data of AIRE expression and function in the periphery in humans, particularly in rare APS 1 patients so that this remains an area of substantial interest. Rare studies in humans of extra-thymic expression have reported AIRE transcript and protein expression in cells of the monocyte/dendritic lineage (13) and in rare non-conventional lymph node APC $(18,21)$. In APS1 patients we (22) and others (17) previously reported peripheral monocyte-derived moDC hyperactivation indicating that AIRE may also play a role in peripheral $\mathrm{T}$-cell activation and thus in tolerance induction in humans.

DC are critical in the initiation of immune responses to invading organisms as well as induction of central and peripheral tolerance to self-antigens (23). Under steady state, DC 
1 display an immature phenotype that correlates with low expression of costimulatory

2 molecules and weak T-cell stimulating properties. Recognition of invading pathogens or local

3 inflammation induces DC differentiation and maturation, characterized by upregulation of

4 major histocompatibility (MHC) antigens (signal I), costimulatory molecules (signal II) on the

5 cell surface and cytokine production (signal III), which are all necessary for the optimal

6 antigen-specific activation of naïve T-cells. However, presentation of antigen with inadequate

7 or alternative co-stimulation and cytokine production will result in T-cell silencing, deletion

8 or T regulatory cell induction. Thus, the pattern of co-stimulation and cytokine production is a

9 central feature distinguishing immunogenic (inflammatory) and tolerogenic DC $(24,25)$ and the differences have been confirmed by distinct mRNA microarray profiles (26). TolDC can be generated in vitro by a variety of methods which include pharmacologic, biologic and genetic engineering (27) and have been generated and applied for therapeutic procedures (25). To better define the role of AIRE in the periphery in humans, we investigated AIRE expression and function in a model of monocyte-derived (mo)DC differentiated into tolerogenic (tolDC) and inflammatory (inflDC) and from healthy controls and APS1 patients. We confirm AIRE expression in moDCs and demonstrate its up-regulation during differentiation. We show for the first time that: 1) tolDCs from healthy individuals express AIRE; 2) that AIRE expression is greater in tolDC than inflDC; 3) that tolDC can also be generated from APECED monocytes and that 4) tolDCs lacking AIRE retain the same phenotype and in vitro function (i.e. high IL-10 production, low T-cell stimulatory capacity).

\section{Patients and Methods:}

$\underline{\text { APS1 patient and healthy control samples }}$

This study included seven APS1 patients, all with confirmed AIRE mutations, 5 males and 2 females aged 3-22 years. All patients were homozygous for the $13 \mathrm{bp}$ deletion in exon 8 
1 c.967_979del13, p.(Leu323fs) which is most frequent in the UK (Pearce et al. 1998), apart

2 from one patient (Pt7) who was a compound heterozygote for c.967_979del13 and c.977C>T.

3 All patients suffered with $\mathrm{CMC}$, mostly mild as well as hypoparathyroidism, adrenal

4 insufficiency and hypothyroidism. Patient 5 also had severe autoimmune hepatitis and

5 malabsorbtion while her brother (patient 6) had only mild CMC at the time of sampling but

6 later developed autoimmune hepatitis (Table 1). Healthy controls were volunteers or anonymous donors from the local blood bank and children undergoing general anaesthesia for non-infectious surgical procedures (ingrown toenails, circumcision etc). Ethical approval was obtained from the Newcastle and North Tyneside Local Research Ethics Committee under the Great North Biobank (GNB) (REC No 10/H0906/22) and the Newcastle Autoimmune Inflammatory Rheumatic Diseases Research (NAIRD) Biobank, (REF No DL-02).

\section{$\underline{\text { Isolation and culture of primary cells }}$}

Peripheral blood mononuclear cells (PBMC) were isolated by density centrifugation from peripheral blood or buffy cones of healthy controls and APS1 patients. Monocytes were positively selected from PBMC using anti-CD14 microbeads (Miltenyi Biotec). CD4 T-cells were enriched using CD4 RosetteSep (StemCell).

\section{Generation of monocyte derived DC}

Monocyte derived DC (moDC) were differentiated as previously described (28). Briefly, monocytes were cultured at $0.5 \times 10^{6}$ cells $/ \mathrm{ml}$ in the presence of IL-4 and GM-CSF $(50 \mathrm{ng} / \mathrm{ml}$ each) (Miltenyi Biotec) for 3 days. On day 3, cells were differentiated into inflammatory (inflDC) by addition of IL-4 and GM-CSF (50 ng/ml each) or into tolerogenic DC (tolDC) by addition of IL-4, GM-CSF (50 ng/ml each) and Dexamethasone (10-6 M, Sigma- Aldrich). On Day 6, all DC were matured by addition of LPS $(0.1 \mu \mathrm{g} / \mathrm{mL})$ (Sigma-Aldrich) for $24 \mathrm{~h}$; tolDC 
1 were additionally treated with addition of dexamethasone (Dex) $\left(10^{-6} \mathrm{M}\right)$ and calcitriol

2 (VitD3) $\left(10^{-10} \mathrm{M}\right)$ (Tocris Bioscience). Cell maturation and differentiation was confirmed by

3 cell surface marker expression and cytokine production. All DC populations were harvested

4 on day 7 and washed extensively before use. Cells were cultured in RPMI 1640, 5 supplemented with $10 \%$ FCS, $2 \mathrm{mM}$ glutamine, $100 \mathrm{U} / \mathrm{ml}$ penicillin and $100 \mu \mathrm{g} / \mathrm{ml}$ 6 streptomycin (RF10) at $37^{\circ} \mathrm{C}$ with $5 \% \mathrm{CO}_{2}$.

7

$\underline{\mathrm{DC}}$ and T-cell cytokine production and proliferation

Supernatants were harvested $24 \mathrm{~h}$ after moDC maturation and stored at $-20^{\circ} \mathrm{C}$. For mixed lymphocyte reaction (MLR), moDC $\left(1 \times 10^{4}\right)$ were cultured with $1 \times 10^{5}$ allogeneic CD4+ Tcells. Supernatants were harvested after 6 days and stored at $-20^{\circ} \mathrm{C}$. Proliferation was assessed by incorporation of $3 \mathrm{H}$-thymidine for the last $8 \mathrm{~h}$ of culture by scintillation counting (Microbeta TriLux, Perkin Elmer). Cytokines in supernatants (IL-12p70, IL-10, TNF- $\alpha$, IFN$\gamma)$ were quantified using sandwich ELISA (BD Biosciences).

$\underline{\text { Flow cytometry }}$

Cell surface expression was investigated using the following anti-human antibodies: HLADR/DP/DQ FITC (Tu39), CD83 Allophycocyanin (HB15e), CD274 (PDL-1) FITC (MIH1) (all BD Pharmingen), CD86 V450 (FUN-1) (BD Horizon) and TLR-2 FITC (TL2.1) (eBioscience). Cells were acquired on a BD FACSCanto II and analyzed using FlowJo software (Treestar).

\section{$\underline{\text { Western blotting (WB) }}$}

Proteins were extracted from cells using RIPA buffer. Equal amounts of proteins were separated by sodium dodecyl sulfate -polyacrylamide gel electrophoresis (SDS-PAGE) using 
$1 \quad 12.5 \%$ polyacrylamide gel, transferred to polyvinylidene difluoride membranes, and blocked

2 in 5\% milk powder/ $0.05 \%$ Tween in Tris-buffered salt solution. Membranes were then

3 probed with antibodies against AIRE (ab82493) (Abcam) and GAPDH (4134) (Cell

4 Signalling) overnight at $4^{\circ} \mathrm{C}$, followed by HRP-conjugated goat anti-rabbit antibody (Dako,

5 P0448). Proteins were visualized using the Immobilon Western detection system (Millipore).

6 AIRE in moDC was identified using human thymus tissue lysate (ab30146) (Abcam) as a

7 positive control.

8

9

\section{$\underline{\text { Gene expression PCR }}$}

Total RNA was isolated from moDC using RNeasy plus micro kit (Qiagen), according to manufacturer instruction. Human thymic total RNA (Clontech) was used as a positive control. cDNA was reverse transcribed from 430ng total RNA using SuperScript III (Invitrogen), according to manufacturer instruction. cDNA was analysed using semi-quantitative PCR with Phire Hot Start II DNA polymerase (Thermo Scientific) according to manufacturer protocol. The following primers were used: AIRE (Fwd 5'AGCAGCCCTGACTCCAAG, Rev 5' CCAACCTGGATGCACTTCTT) and GAPDH (Fwd GTGAACCATGAGAAGTATGACAAC, Rev 5' CATGAGTCCTTCCACGATACC ) under the following cycling conditions: 30 s at $98^{\circ} \mathrm{C}, 36$ cycles for AIRE or 22 cycles for GAPDH of $98^{\circ} \mathrm{C}$ for $5 \mathrm{~s}, 60^{\circ} \mathrm{C}$ for $5 \mathrm{~s}, 72^{\circ} \mathrm{C}$ for $30 \mathrm{~s}$ followed by $2 \mathrm{~m}$ at $72^{\circ} \mathrm{C}$. PCR products were visualized using $1 \%$ agarose electrophoresis.

\section{$\underline{\text { Statistical analysis and data plotting }}$}

Densitometric analysis was performed using ImageJ software. For analysis of AIRE gene and protein expression, the bands on each agarose gel or western blot were normalized to the 
1 intensity of the day (D) 7 inflDC sample. Statistical analysis of data was performed with

2 GraphPad Prism 5.0 using ANOVA with Sidak's or Tukey's comparison tests.

3

4

Results:

AIRE is expressed in monocyte-derived dendritic cells

While AIRE gene and protein expression in mice was confirmed in lymph node eTACs, (18), there is conflicting evidence as to the expression of AIRE protein in monocyte-DC lineages. Using thymic RNA as a positive control and site specific primers against the canonical AIRE RNA transcript sequence (NM_000383), we first assessed AIRE gene expression (mRNA). We confirmed the AIRE transcript in monocytes and moDC (Figure 1A) and contrary to previous findings (12) demonstrated AIRE mRNA expression to be highest in CD14+ monocytes (D0), with a modest decrease during moDC differentiation although none of the changes were statistically significant. In matured DC we confirmed that AIRE is expressed in inflDC and report for the first time that tolDC also express AIRE mRNA. This expression is somewhat higher albeit not significantly different compared to inflDC. We next identified very weak AIRE protein expression in moDC (Figure 1B) and show in healthy controls that AIRE protein levels increased slightly in mature tolDC and inflDCs compared to CD14+ monocytes (D0) where protein was undetectable. The level of mRNA transcript did not correlate with protein expression, and there seemed to be an inverse correlation in that CD14+ monocyte expressed highest levels of mRNA but no protein was detectable. We did not attempt AIRE mRNA and protein detection in patients with AIRE deficiency as we do not expect to find transcript and protein from a non-functional gene.

TolDC can be generated from APS1 patients and retain their tolerogenic phenotype and function 
1 To assess whether AIRE has a role in the generation and function of tolDC, we differentiated

2 inflDC and tolDC from APS1 patients and assessed moDC phenotype and function. Our

3 results demonstrate that APS1 tolDC exhibit the same characteristics as tolDCs from healthy

4 individuals in that they maintain high MHC class II expression, have low CD83 and CD86

5 expression, higher TLR2 expression and lower PD-L1 expression than their inflDC

6 counterpart (Figure 2A and 2B). Equally, the differences seen in these markers between

7 inflDC and tolDCs are comparable in healthy controls and APS1 patients. Similar to tolDC

8 from healthy individuals, APS1 tolDC secrete high levels of IL-10 (Figure 3A). Levels of

9 TNF $\alpha$ produced by both inflDC and tolDC in both studied groups were comparable while IL-

12 production was negligible. APS1 tolDC induced sub-optimal T-cell proliferation (Figure 3B) in a mixed lymphocyte reaction but this did not differ significantly from levels induced by APS1 inflDCs, as opposed to tolDC generated from healthy individuals. Equally, when APS1 tolDC are co-cultured with healthy CD4 T-cells, lower levels of IFN- $\gamma$ and higher levels of IL-10 are detected compared to inflDC (Figure 3C) and these values are comparable between healthy controls and APS1 patients. While the generation of InflDC from APS1 patient monocytes has been reported previously (22), we now show that tolDC can also be generated from APS1 monocytes. These data demonstrate that the functional features of APS1 tolDC (i.e. high IL-10 production, low T-cell stimulatory capacity) are comparable to healthy controls, indicating that AIRE is not required for the differentiation or for the immunosuppressive function of tolDC.

\section{Discussion and Conclusions:}

Although the presence and role of AIRE in thymic medullary epithelial cells (MEC) in both mice and humans is undisputed, the expression and putative function of AIRE in the periphery remains controversial $(4,18)$. While there are several reports confirming AIRE 
1 protein expression in human thymic DC (29), the consistency of AIRE protein presence in

2 peripheral human DC is a matter of debate (15). Our results confirm previous reports of AIRE transcript (mRNA) expression in stimulated moDC (12). We were also able to detect AIRE at the protein level albeit in very low quantities. When compared to equal quantities of thymic lysate, it is clear that the expression of AIRE in moDC is substantially lower and consistent with previous reports of very low/undetectable AIRE protein expression in other immune cells in the periphery (13). Because of the low expression of AIRE in moDCs our attempts to silence AIRE in these cells were not reliable (data not shown). We accept that the very low protein levels detected question the implication of the finding and we acknowledge that similarly low levels previously reported underpin controversies about its biological significance, if any. However, band intensities differ and are higher in tolDCs than in their inflammatory counterpart. In CD14+ monocytes, we detected AIRE transcript which was actually at its highest level compared to other moDC maturation stages but this did not correlate with protein expression which was absent, consistent with previous reported findings of this discrepancy in peripheral cells. Nevertheless, even though we cannot convincingly demonstrate AIRE protein in DC from healthy controls, we report for the first time that tolerogenic DCs generated from monocytes isolated from AIRE-deficient patients are comparable to those generated from healthy controls. Consequently, AIRE is unlikely to play a role in tolDC generation or in regulating their typical immunosuppressive properties (e.g. high IL-10 production, low T-cell stimulatory capacity). Having said that, we only investigated the T-cell stimulatory function of tolDC in an allogeneic setting; it would be interesting for future studies to use an antigen-specific model so that the antigen-processing function of tolDC in AIRE-deficient APS-1 patients can be assessed as well.

AIREs putative role in the periphery remains enigmatic. In mice, transcription of genes that are under Aire control in the thymus differs to those in the periphery (30). AIRE 
expressing APC have been reported to control immune cell function via antigen-specific Tcell suppression $(18,31)$. Studies in AIRE-/- mice suggest a role for AIRE-expressing DC in the induction of T-cell tolerance, independent from central tolerance $((17,20)$. Several early reports suggest a role for AIRE in the induction of T-regulatory (Treg) cells in the periphery $(32,33)$, while a recent report demonstrates that Aire promotes the perinatal generation of a distinct compartment of Foxp3+CD4+ T (Treg) cells, which stably persists in adult mice (19).

The few reports investigating AIRE function in peripheral cells of APS1 patients agree with mice studies showing deregulated DC function and support a role for AIRE in peripheral tolerance. The eTACs from mice have also been identified in human peripheral lymphoid tissue (18), yet their tolerogenic function still remains to be confirmed. This is a first study of AIRE in human tolerogenic DC and shows that AIRE expression is higher in these cells than in their inflammatory counterpart. However, assessment of typical features of tolDC (i.e. high IL-10 production, low T-cell stimulatory capacity) in APS1 patients did not confirm a role for AIRE in this subset of cells, in contrast to other reports of AIRE expressing cells in the periphery, albeit in mice $(15,18)$.

There is much intrigue in the similarities, or lack of, between Aire ${ }^{-/-}$mouse models and human APS1. Aire ${ }^{-/-}$mouse models share an autoimmune phenotype with APS1 patients, although mice mostly do not develop symptoms or have very mild disease depending on the genetic background $(20,34)$. Importantly, Aire-/- mice develop autoantibodies to a different set of self-antigens compared to human patients (35). In mice, autoantibodies to IFN type I and Th-17 cytokines have only been reported in aged Air $e^{-/-}$mice on a BALB/c background (36), yet these mice do not succumb to CMC, These findings may suggest that Aire controls an alternative set of genes in humans and mice (35) and advocates caution in extrapolating findings from murine studies to human disease. 
Although we demonstrate that AIRE is not essential for the generation or function of tolerogenic moDC, we do not discount a role for AIRE in peripheral tolerance. In humans a distinct DC subset of inflammatory (infl) DC has recently been described which differentiates from blood monocytes recruited to the site of inflammation (37) and is the likely in vivo analogue of the in vitro generated moDC. In vitro generated inflDC present antigens, activate CD4 T-cells and guide cytokine responses depending on environmental cues while tolDCs induce tolerance through altered presentation of antigen to T-cells and through the production of suppressive cytokines that inhibit effect T-cell activation $(25,38)$. As such, moDC/tolDC were an interesting target to study AIRE and the fact that a role in generation and function was not found by no means excludes a role for AIRE in other subsets of tolerogenic DC in the periphery, particularly those subsets where AIRE expression has been documented (10). It is also possible that AIRE -/- differ from AIRE +/+ tolDCs in ways which were not assessed in this study such as their transcriptome profile or that AIRE expression may be relevant for function of other (non-DC) cell types in the periphery as was found in mice (18). To understand the role of AIRE in the periphery, it is crucial to define how AIRE functions beyond the expression of ectopic genes in the mTECs as recent data indicate that in the periphery, AIRE may regulate a large number of genes with different functions $(12,39,40)$. Unexpectedly, it was recently shown that certain heterozygous AIRE mutations may play a hitherto unrecognised role in common organ-specific autoimmune diseases, suggesting that our current understanding of its function is still limited (41). In conclusion, our findings suggest that AIRE does not have a role in the induction and function of monocyte-derived tolerogenic DC in humans, but these findings do not exclude a role for AIRE in in peripheral tolerance mediated by other cell types.

\section{Declaration of Interest}


1 The authors report no conflicts of interests. The study was funded by the JGW Patterson

2 Foundation.

3 Author contribution

4 DL and CMUH designed the study; KLC, DL and CMUH wrote the manuscript. KLC 5 performed the experiments. MA, PDA, TDC, SHP contributed patient samples and reviewed 6 the manuscript. 


\section{References}

1. Anderson, M. S., E. S. Venanzi, L. Klein, Z. Chen, S. P. Berzins, S. J. Turley, H. von Boehmer, R. Bronson, A. Dierich, C. Benoist, and D. Mathis. 2002. Projection of an immunological self shadow within the thymus by the aire protein. Science 298: 13951401.

2. Ruan, Q. G., K. Tung, D. Eisenman, Y. Setiady, S. Eckenrode, B. Yi, S. Purohit, W. P. Zheng, Y. Zhang, L. Peltonen, and J. X. She. 2007. The autoimmune regulator directly controls the expression of genes critical for thymic epithelial function. Journal of immunology 178: 7173-7180.

3. Cheng, M. H., and M. S. Anderson. 2012. Monogenic autoimmunity. Annual review of immunology 30: 393-427.

4. Mathis, D., and C. Benoist. 2009. Aire. Annual review of immunology 27: 287-312.

5. Pearce, S. H., T. Cheetham, H. Imrie, B. Vaidya, N. D. Barnes, R. W. Bilous, D. Carr, K. Meeran, N. J. Shaw, C. S. Smith, A. D. Toft, G. Williams, and P. Kendall-Taylor. 1998. A common and recurrent 13-bp deletion in the autoimmune regulator gene in British kindreds with autoimmune polyendocrinopathy type 1. American journal of human genetics 63: 1675-1684.

6. Puel, A., R. Doffinger, A. Natividad, M. Chrabieh, G. Barcenas-Morales, C. Picard, A. Cobat, M. Ouachee-Chardin, A. Toulon, J. Bustamante, S. Al-Muhsen, M. Al-Owain, P. D. Arkwright, C. Costigan, V. McConnell, A. J. Cant, M. Abinun, M. Polak, P. F. Bougneres, D. Kumararatne, L. Marodi, A. Nahum, C. Roifman, S. Blanche, A. Fischer, C. Bodemer, L. Abel, D. Lilic, and J. L. Casanova. 2010. Autoantibodies against IL-17A, IL-17F, and IL-22 in patients with chronic mucocutaneous candidiasis and autoimmune polyendocrine syndrome type I. The Journal of experimental medicine 207: 291-297. 
17 Kisand, K., A. S. Boe Wolff, K. T. Podkrajsek, L. Tserel, M. Link, K. V. Kisand, E. Ersvaer, J. Perheentupa, M. M. Erichsen, N. Bratanic, A. Meloni, F. Cetani, R. Perniola, B. Ergun-Longmire, N. Maclaren, K. J. Krohn, M. Pura, B. Schalke, P. Strobel, M. I. Leite, T. Battelino, E. S. Husebye, P. Peterson, N. Willcox, and A. Meager. 2010. Chronic mucocutaneous candidiasis in APECED or thymoma patients correlates with autoimmunity to Th17-associated cytokines. The Journal of experimental medicine 207: 299-308.

8. Kisand, K., D. Lilic, J. L. Casanova, P. Peterson, A. Meager, and N. Willcox. 2011. Mucocutaneous candidiasis and autoimmunity against cytokines in APECED and thymoma patients: clinical and pathogenetic implications. European journal of immunology 41: 1517-1527.

9. Nagamine, K., P. Peterson, H. S. Scott, J. Kudoh, S. Minoshima, M. Heino, K. J. E. Krohn, M. D. Lalioti, P. E. Mullis, S. E. Antonarakis, K. Kawasaki, S. Asakawa, F. Ito, and N. Shimizu. 1997. Positional cloning of the APECED gene. Nat Genet 17: 393-398.

10. Poliani, P. L., K. Kisand, V. Marrella, M. Ravanini, L. D. Notarangelo, A. Villa, P. Peterson, and F. Facchetti. 2010. Human Peripheral Lymphoid Tissues Contain Autoimmune Regulator-Expressing Dendritic Cells. Am J Pathol 176: 1104-1112.

11. Suzuki, E., Y. Kobayashi, O. Kawano, K. Endo, H. Haneda, H. Yukiue, H. Sasaki, M. Yano, M. Maeda, and Y. Fujii. 2008. Expression of AIRE in thymocytes and peripheral lymphocytes. Autoimmunity 41: 133-139.

12. Sillanpaa, N., C. G. Magureanu, A. Murumagi, A. Reinikainen, A. West, A. Manninen, M. Lahti, A. Ranki, K. Saksela, K. Krohn, R. Lahesmaa, and P. Peterson. 2004. Autoimmune regulator induced changes in the gene expression profile of human monocyte-dendritic cell-lineage. Mol Immunol 41: 1185-1198. 
1 13. Kogawa, K., S. Nagafuchi, H. Katsuta, J. Kudoh, S. Tamiya, Y. Sakai, N. Shimizu, and M. Harada. 2002. Expression of AIRE gene in peripheral monocyte/dendritic cell lineage. Immunol Lett 80: 195-198.

14. Pontynen, N., M. Strengell, N. Sillanpaa, J. Saharinen, I. Ulmanen, I. Julkunen, and L. Peltonen. 2008. Critical immunological pathways are downregulated in APECED patient dendritic cells. J Mol Med 86: 1139-1152.

15. Eldershaw, S. A., D. M. Sansom, and P. Narendran. 2011. Expression and function of the autoimmune regulator (Aire) gene in non-thymic tissue. Clin Exp Immunol 163: 296-308.

16. Kisand, K., and P. Peterson. 2015. Autoimmune Polyendocrinopathy Candidiasis Ectodermal Dystrophy. Journal of clinical immunology.

17. Ramsey, C., S. Hassler, P. Marits, O. Kampe, C. A. Surh, L. Peltonen, and O. Winqvist. 2006. Increased antigen presenting cell-mediated $\mathrm{T}$ cell activation in mice and patients without the autoimmune regulator. Eur J Immunol 36: 305-317.

18. Gardner, J. M., T. C. Metzger, E. J. McMahon, B. B. Au-Yeung, A. K. Krawisz, W. Lu, J. D. Price, K. P. Johannes, A. T. Satpathy, K. M. Murphy, K. V. Tarbell, A. Weiss, and M. S. Anderson. 2013. Extrathymic Aire-expressing cells are a distinct bone marrow-derived population that induce functional inactivation of CD4(+) T cells. Immunity 39: 560-572.

19. Yang, S., N. Fujikado, D. Kolodin, C. Benoist, and D. Mathis. 2015. Regulatory T cells generated early in life play a distinct role in maintaining self-tolerance. Science.

20. Hubert, F. X., S. A. Kinkel, P. E. Crewther, P. Z. F. Cannon, K. E. Webster, M. Link, R. Uibo, M. K. O'Bryan, A. Meager, S. P. Forehan, G. K. Smyth, L. Mittaz, S. E. Antonarakis, P. Peterson, W. R. Heath, and H. S. Scott. 2009. Aire-Deficient C57BL/6 
Mice Mimicking the Common Human 13-Base Pair Deletion Mutation Present with Only a Mild Autoimmune Phenotype. Journal of immunology 182: 3902-3918.

21. Poliani, P. L., K. Kisand, V. Marrella, M. Ravanini, L. D. Notarangelo, A. Villa, P. Peterson, and F. Facchetti. 2010. Human peripheral lymphoid tissues contain autoimmune regulator-expressing dendritic cells. The American journal of pathology 176: $1104-1112$.

22. Ryan, K. R., M. Hong, P. D. Arkwright, A. R. Gennery, C. Costigan, M. Dominguez, D. Denning, V. McConnell, A. J. Cant, M. Abinun, G. P. Spickett, and D. Lilic. 2008. Impaired dendritic cell maturation and cytokine production in patients with chronic mucocutanous candidiasis with or without APECED. Clin Exp Immunol 154: 406-414.

23. Banchereau, J., and R. M. Steinman. 1998. Dendritic cells and the control of immunity. Nature 392: 245-252.

24. Hubo, M., B. Trinschek, F. Kryczanowsky, A. Tuettenberg, K. Steinbrink, and H. Jonuleit. 2013. Costimulatory molecules on immunogenic versus tolerogenic human dendritic cells. Frontiers in immunology 4: 82.

25. Hilkens, C. M., J. D. Isaacs, and A. W. Thomson. 2010. Development of dendritic cell-based immunotherapy for autoimmunity. International reviews of immunology 29: 156-183.

26. Suciu-Foca Cortesini, N., F. Piazza, E. Ho, R. Ciubotariu, J. LeMaoult, R. DallaFavera, and R. Cortesini. 2001. Distinct mRNA microarray profiles of tolerogenic dendritic cells. Human immunology 62: 1065-1072.

27. Coates, P. T., B. L. Colvin, K. Kaneko, T. Taner, and A. W. Thomson. 2003. Pharmacologic, biologic, and genetic engineering approaches to potentiation of donorderived dendritic cell tolerogenicity. Transplantation 75: 32S-36S. 
1 28. Anderson, A. E., D. J. Swan, B. L. Sayers, R. A. Harry, A. M. Patterson, A. von

Delwig, J. H. Robinson, J. D. Isaacs, and C. M. Hilkens. 2009. LPS activation is required for migratory activity and antigen presentation by tolerogenic dendritic cells. Journal of leukocyte biology 85: 243-250.

29. Heino, M., P. Peterson, J. Kudoh, K. Nagamine, A. Lagerstedt, V. Ovod, A. Ranki, I. Rantala, M. Nieminen, J. Tuukkanen, H. S. Scott, S. E. Antonarakis, N. Shimizu, and K. Krohn. 1999. Autoimmune regulator is expressed in the cells regulating immune tolerance in thymus medulla. Biochemical and biophysical research communications 257: 821-825.

30. Schaller, C. E., C. L. Wang, G. Beck-Engeser, L. Goss, H. S. Scott, M. S. Anderson, and M. Wabl. 2008. Expression of Aire and the early wave of apoptosis in spermatogenesis. Journal of immunology 180: 1338-1343.

31. Grupillo, M., G. Gualtierotti, J. He, G. Sisino, R. Bottino, W. A. Rudert, M. Trucco, and Y. Fan. 2012. Essential roles of insulin expression in Aire(+) tolerogenic dendritic cells in maintaining peripheral self-tolerance of islet beta-cells. Cell Immunol 273: 115-123.

32. Ryan, K. R., C. A. Lawson, A. R. Lorenzi, P. D. Arkwright, J. D. Isaacs, and D. Lilic. 2005. CD4+CD25+ T-regulatory cells are decreased in patients with autoimmune polyendocrinopathy candidiasis ectodermal dystrophy. The Journal of allergy and clinical immunology 116: 1158-1159.

33. Kekalainen, E., H. Tuovinen, J. Joensuu, M. Gylling, R. Franssila, N. Pontynen, K. Talvensaari, J. Perheentupa, A. Miettinen, and T. P. Arstila. 2007. A defect of regulatory $\mathrm{T}$ cells in patients with autoimmune polyendocrinopathy-candidiasisectodermal dystrophy. Journal of immunology 178: 1208-1215. 
1 34. Jiang, W. Y., M. S. Anderson, R. Bronson, D. Mathis, and C. Benoist. 2005. Modifier loci condition autoimmunity provoked by Aire deficiency. Journal of Experimental Medicine 202: 805-815.

35. Pontynen, N., A. Miettinen, T. P. Arstila, O. Kampe, M. Alimohammadi, O. Vaarala, L. Peltonen, and I. Ulmanen. 2006. Aire deficient mice do not develop the same profile of tissue-specific autoantibodies as APECED patients. $J$ Autoimmun 27: 96104.

36. Karner, J., A. Meager, M. Laan, J. Maslovskaja, M. Pihlap, A. Remm, E. Juronen, A. S. B. Wolff, E. S. Husebye, K. T. Podkrajsek, N. Bratanic, T. Battelino, N. Willcox, P. Peterson, and K. Kisand. 2013. Anti-cytokine autoantibodies suggest pathogenetic links with autoimmune regulator deficiency in humans and mice. Clin Exp Immunol 171: $263-272$.

37. Segura, E., M. Touzot, A. Bohineust, A. Cappuccio, G. Chiocchia, A. Hosmalin, M. Dalod, V. Soumelis, and S. Amigorena. 2013. Human inflammatory dendritic cells induce Th17 cell differentiation. Immunity 38: 336-348.

38. Segura, E., and S. Amigorena. 2013. Inflammatory dendritic cells in mice and humans. Trends in immunology 34: 440-445.

39. Macedo, C., A. F. Evangelista, M. M. Marques, S. Octacilio-Silva, E. A. Donadi, E. T. Sakamoto-Hojo, and G. A. Passos. 2013. Autoimmune regulator (Aire) controls the expression of microRNAs in medullary thymic epithelial cells. Immunobiology 218 : 554-560.

40. Zhu, W. F., W. Yang, Z. F. He, X. Y. Liao, J. Wu, J. T. Sun, Y. Yang, and Y. Li. 2011. Overexpressing autoimmune regulator regulates the expression of toll-like receptors by interacting with their promoters in RAW264.7 cells. Cell Immunol 270: 156-163. 
1 41. Oftedal, B. E., A. Hellesen, M. M. Erichsen, E. Bratland, A. Vardi, J. Perheentupa, E. H. Kemp, T. Fiskerstrand, M. K. Viken, A. P. Weetman, S. J. Fleishman, S. Banka, W. G. Newman, W. A. Sewell, L. S. Sozaeva, T. Zayats, K. Haugarvoll, E. M. Orlova, J. Haavik, S. Johansson, P. M. Knappskog, K. Lovas, A. S. Wolff, J. Abramson, and E. S. Husebye. 2015. Dominant Mutations in the Autoimmune Regulator AIRE Are Associated with Common Organ-Specific Autoimmune Diseases. Immunity 42: 11857 1196. 


\section{Figure 1. AIRE is expressed in moDC and is higher in TolDC than in InflDC}

2 Monocytes from healthy controls were cultured with IL-4 / GM-CSF to produce inflDC (Infl)

3 and additionally with Dex for tolDC (Tol). At day 6 moDC were matured with LPS for inflDC

4 and additionally with VitD3 and Dex for tolDC. At the given timepoints, cells were harvested

5 for RNA extraction for (A) PCR or lysed for (B) Western blot analysis. For densitometry analysis, band intensities were first normalised to D7 InflDC for each gene/protein, then AIRE band intensities were normalised to each GAPDH band for every time point. Graphs illustrate the mean + SEM of 5 independent experiments. One-way ANOVA analysis with Sidak's multiple comparison test was performed. $\mathrm{ns}=$ not significant, ${ }^{*} \mathrm{p}<0.05,{ }^{*} \mathrm{p}<0.01$, $* * * \mathrm{p}<0.005, * * * * \mathrm{p}<0.001$

Figure 2. TolDC can be generated from APS1 patient monocytes and have a tolerogenic phenotype

Monocytes from healthy controls or APS1 patients were cultured with IL-4 / GM-CSF to produce inflDC (Infl) and additionally with Dex for tolDC (Tol). At day 6 moDC were matured with LPS for inflDC and additionally with VitD3 and Dex for tolDC. (A) moDC were stained for the cell surface markers (MHC classII, CD83, CD86, TLR-2 and PDL-1) and analyzed by flow cytometry. A comparison of median fluorescence intensity (MFI) between a sample from a healthy control and APS1 patients is shown in (A). In (B) a summary of individual results is shown for all healthy control and patient samples. Graphs illustrate individual values and mean values with number of experiments shown on graph. One-way ANOVA analysis with Tukey's multiple comparison test was performed. ns = not significant, ${ }^{*} \mathrm{p}<0.05,{ }^{*} * \mathrm{p}<0.01, * * * \mathrm{p}<0.005, * * * * \mathrm{p}<0.001$. 
2 Figure 3. TolDC generated from APS1 patient monocytes show cytokine production and

3 a mixed lymphocyte reaction (MLR) consistent with a tolerogenic function. (A) Culture

4 supernatants were taken on D7 and assayed for the cytokines shown, by ELISA. (B) MoDC

5 were co-cultured with allogeneic CD4+ T-cells in a an MLR for 6 days. Proliferation was

6 assessed by $3 \mathrm{H}$ incorporation. (C) MLR culture supernatants were taken at D6 and assayed

7 for the given cytokines, by ELISA. Graphs illustrate individual values and mean values with

8 number of experiments shown on graph. One-way ANOVA analysis with Tukey's multiple

9 comparison test was performed. ns $=$ not significant, ${ }^{*} p<0.05, * * p<0.01, * * * p<0.005$, $* * * * \mathrm{p}<0.001$. 